\title{
DFFB Gene
}

National Cancer Institute

\section{Source}

National Cancer Institute. DFFB Gene. NCI Thesaurus. Code C126525.

This gene is involved in apoptosis. 